\title{
In vitro osteoinduction potential of a novel silica coated hydroxyapatite bioscaffold seeded with rabbit mesenchymal stem cell
}

\begin{abstract}
The objective of this study was to assess cyto compatibility of silica coated hydroxyapatite (HASi) bioceramic and rabbit bone marrow derived mesenchymal stem cells (rBMSC) in terms of cell attachment, cell morphology, cell proliferation and osteogenic differentiation. The rBMSC were seeded on HASi blocks and maintained in standard culture conditions. Samples on day 3 and day 17 post-seeding were subjected for scanning electron microscopy (SEM). Day 3 post-seeding samples showed stem cell attachment with a wavy surface morphology and day 17 post-seeding sample showed expansion of attached rBMSCs with cell sheet like morphology and deposition of matrix in the form of crystals. Alizarin red staining of these trypsinized crystals showed positive staining, indicative of presence of calcium. Histological sections of decalcified samples on day 17 post-seeding showed areas of early new bone formation. It was concluded that silica coated hydroxyapatite could induce the osteogenic differentiation of rBMSC by day 17 post-seeding in the absence of any osteogenic induction media which was confirmed by the presence of crystal like matrix deposition on scanning electron microscopic analysis, alizarin red staining of trypsinized crystals and early new bone formation in histological sections.
\end{abstract}

Keywords: mesenchymal stem cell, bioceramic scaffold, scanning electron microscopy, rabbit, cell seeding, osteoinduction
Volume I Issue 2 - 2016

\begin{abstract}
Ninu Ajantha Ravindran,' Swapan Kumar Maiti, ${ }^{2}$ Sangeetha Palakkara, ${ }^{2}$ Deepika Kritaniya, ${ }^{2}$ Tamil Mahan, ${ }^{\prime}$ Naveen Kumar ${ }^{2}$ 'Department of Veterinary Surgery \& Radiology, Veterinary College \& Research Institute, India

${ }^{2}$ Surgery Division, Indian Veterinary Research Institute, India
\end{abstract}

Correspondence: Swapan Kumar Maiti, Principal Scientist, Surgery Division, Indian Veterinary Research Institute, Izatnagar-243 I22, Uttar-Pradesh, India, Tel +9| 94|2049|69, Email swapanivri@gmail.com

Received: December 17, 2015 | Published: January 29, 2016
Abbreviations: rBMSC, rabbit bone marrow derived mesenchymal stem cells; HASi, silica coated hydroxyapatite; SEM, scanning electron microscopy; CFU-F, colony forming unit-fibroblast; CFU-OB, colony forming unit-osteoblast; OS, osteogenic supplement; PBS, phosphate buffer saline; MSC, mesenchymal stem cell; HMDS, hexamethyl disilazane; DMEM, dulbecco's modified eagle medium; PI, propidium iodine

\section{Introduction}

The knowledge and principles from interconnected disciplines of bioengineering, material science and life sciences are combined in tissue engineering with the aim of developing a construct that can wholly or partly restore/maintain/augment the function of a damaged tissue or organ. Tissue engineering construct generally comprises cells on a suitable scaffold. The ultimate functionality of a tissue engineering construct is mainly dependent on the cell behavior on scaffolds. ${ }^{1,2}$ The proliferation and cell viability on scaffolds varies from one cell type to another and from one species to another. So it is important to investigate individual cell-scaffold combinations. ${ }^{3}$

The similarity in structure and composition to bone mineral, osteoconducive properties, ability to integrate with the bony tissue and absence of immune response makes hydroxyapatite $\left[\mathrm{Ca}_{10}\right.$ $\left.\left(\mathrm{PO}_{4}\right)_{6}(\mathrm{OH})_{2}\right]$ the choice of calcium phosphate biomaterial ${ }^{4,5}$ with only disadvantage of slow rate of resorption. ${ }^{6}$ On contrary, silicacalcium phosphate composite in comparison to calcium phosphate is rich biomaterials having a faster resorption rate. ${ }^{7}$ Studies have shown that coating of hydroxyapatite with a calcium silicate containing layer encourages cell proliferation and osteogenic differentiation of human bone marrow-derived stromal cells. ${ }^{8}$ So in this study a triphasic composite scaffold (calcium silicate, hydroxyapatite and tricalcium phosphate), namely HASi with elements in the following percentages: 66.36\%-calcium, 25.35\%-phosphorus and silicon - $8.29 \%$ and porosity of $50-500 \mu \mathrm{m}$ was used to evaluate the rBMSC proliferation and differentiation properties in vitro.

The present study was therefore, undertaken with the aim of assessing cyto compatibility of HASi and rBMSC in terms of cell attachment, cell morphology, cell proliferation and osteogenic differentiation.

\section{Materials and methods}

\section{rBMSC isolation and culture}

Bone marrow aspiration, isolation and culture of rBMSC were done as per previous reports. ${ }^{9}$ We have used third passage of rBMSC for this study.

\section{Quantification of mesenchymal progenitors (CFU-F assay)}

For CFU-F assay, $1 \times 106$ bone marrow nucleated cells per $60-\mathrm{mm}$ dish were seeded and cultured at $37^{\circ} \mathrm{C}$ in $5 \% \mathrm{CO}_{2}$ for 12 days with media change on every 2 days, followed by crystal violet staining. The numbers of CFU-Fs were counted under a microscope.

\section{Quantification of osteoblasts (CFU-OB assay)}

For CFU-OB assay, $1 \times 106$ bone marrow nucleated cells per $60-\mathrm{mm}$ dish were seeded and cultured for 21 days in 
differentiation medium ( $\alpha$-MEM containing $10 \%$ FBS, $1 \%$ penicillin/streptomycin, $50 \mu \mathrm{g} / \mathrm{ml}$ L-ascorbic acid and $2.0 \mathrm{mM}$ b-glycerophosphate). Media was changed on every 2 days. Alizarin red staining was done to identify the mineralized bone matrix of osteoblast differentiated colonies, designated as CFU-OB colonies.

\section{Osteogenic differentiation}

Undifferentiated cells were induced towards the osteogenic lineage. Putative MSC was seeded in six well plates at a density of approximately 3000 cells $/ \mathrm{cm}^{2}$ and cultured in expansion media till it reached $90-100 \%$ confluence. Osteogenic differentiation was induced by culturing the cells for 20days in osteogenic induction medium (OS) consisting of $100 \mathrm{nM}$ dexamethasone, $10 \mathrm{mM} \beta$-glycerophosphate, and $0.05 \mathrm{mM}$ L-ascorbic acid-2-phosphate and 10\% FBS in low glucose DMEM. As a negative control an equal number of wells were maintained in expansion media for 20days. The media in both groups was replaced completely every 4 days. The cells were cultured at $37^{\circ} \mathrm{C}$ in a $\mathrm{CO}_{2}$ incubator at $5 \% \mathrm{CO}_{2}$ and maximum humidified environment. The presence/differentiation of osteoblasts was confirmed by mineralization staining (Alizarin red S staining) and alkaline phosphatase (ALP) activity.

\section{Fluorescent staining of rBMSC-scaffold constructs}

For assessment of cell viability and attachment on scaffold, the cell-scaffold constructs were rinsed twice with sterile PBS and submersed in $3 \mathrm{~mL}$ of PBS. CFDA-SE (final concentration $2.5 \mathrm{mM}$ ) was then added to the PBS and incubated for 15 minutes at $37^{\circ} \mathrm{C}$ to label live cells green (emission at $517 \mathrm{~nm}$ ) by labeling intracellular esterase as recommended by the manufacturer. They were rinsed twice again with PBS. Propidium iodide (PI) $(20 \mu \mathrm{g} / \mathrm{ml})$.

(Molecular Probes) in PBS was added for 2 min at room temperature followed by washing with PBS. All steps were performed in the dark and samples were then viewed under a fluorescence microscope.

\section{Scanning electron microscopy (SEM) study}

Attachment properties and morphology of rBMSCs from third passage were assessed on HASi blocks of $0.3 \times 0.2 \times 0.1 \mathrm{~cm}$ dimension. For this, the scaffold blocks were coated with fibronectin for $24 \mathrm{~h}$ and then surface seeding of third passage rBMSCs was done with $1 \times 10^{6}$ cells per $\mathrm{mL}$ of DMEM (Gibco) medium containing $10 \%$ fetal bovine serum (Gibco) and antibiotics (penicillin $\mathrm{G}, 100 \mathrm{U} / \mathrm{ml}$; streptomycin, $0.1 \mathrm{mg} / \mathrm{ml}$; amphotericin B, $0.25 \mu \mathrm{g} / \mathrm{ml}$ ) in six well plates followed by incubation at $37^{\circ} \mathrm{C}$ in a humidified $5 \% \mathrm{CO}_{2}$ environment. Unseeded scaffolds were also maintained in the same culture conditions as control. The cell-loaded scaffolds and control were rinsed with PBS after 3 and 17days of cell seeding respectively, and fixed with glutaraldehyde $2.5 \%$ for $1 \mathrm{~h}$. Then the scaffolds were dehydrated with a graded ethanol series $(30 \%, 50 \%, 70 \%, 90 \%$ and $100 \%)$ and incubated for 10 minutes in hexamethyl-disilazane (HMDS) and dried overnight in a desiccators, and were gold coated. They were observed under scanning electron microscope (Jeol, Japan Electron Opticals Limited, JSM-6610LV-3 lakh magnification).

\section{Evaluation of calcium crystals}

The ability of cells to produce mineralized matrix and calcium crystals is a key factor for bone regeneration. To determine the formation of calcified extracellular matrix deposition due to mineralization phenomena, a histochemical technique was used.
Cells harvested from trypsinized scaffolds were fixed in $70 \%$ ethanol followed by washing with PBS and stained with $1 \%$ alizarin red solution for $2 \mathrm{~min}$. They were washed again with PBS and observed under a light microscope.

\section{Histological evaluation}

The decalcified scaffolds with Goodling Stewart's solution after day 17 post-seeding maintained in the same culture conditions as for SEM analysis. They were subjected for histological study with H \& E staining to evaluate the bone formation ability.

\section{Statistical analysis}

All the data were analyzed by analysis of variance (ANOVA) as per standard statistical methods, using SPSS software package (version 16).

\section{Results}

Bone marrow collection from rabbits was carried out as per the standardized procedure. The mesenchymal stem cells were successfully isolated, cultured and propagated from all the aspirated bone marrow samples. Third passage of rBMSC was used for seeding in HASi scaffold (Figure 1A) From CFU-F assay, $52 \pm 2.3$ (mean \pm SD) colonies of fibroblast were obtained from three different trials and from CFU-OB assay, $49 \pm 2.6$ (mean $\pm \mathrm{SD}$ ) colonies of osteoblasts were obtained (Figure 1B) from an initial seeding density of $1 \times 10^{6}$ cells from buffy coat and third passage respectively in $60 \mathrm{~mm}$ culture dish. The comparative histogram showing the mean $\pm \mathrm{SD}$ is shown in (Figure 1C).

To characterize unique differentiating property of rBMSCs and mineral deposition, osteogenic differentiation was performed in monolayer culture for 3 weeks. The cells changed from a fibroblastic appearance to a more cuboidal appearance at day 9 of culture. Proliferation and differentiation of control and OS cultures were compared using inverted microscope. In both cultures, cells proliferated and reached almost complete confluence at day 9. In OS cultures, nodular aggregates of cells became evident at day 9 of culture and increased up to 21 days. These aggregates were characterized by deposits of amorphous material. In control cultures aggregates were not observed and deposits were also lacking. The OS cultures were stained positive (orange red) with alizarin red $\mathrm{S}$ stain for mineral (calcium) deposition in their newly formed matrix (Figure 1D). Alizarin red positive nodular aggregates present at day 21 were larger and stained more intensively, indicating that a more extensive calcium deposition had occurred. In the control wells, adherent monolayer of swirling spindle shaped cells became over confluent without any cell aggregates and was stained negative for mineral deposition. ALP activity or expression was higher in osteogenic differentiated cells (Figure1E) than control culture (Figure 1F). In positive cases, the nucleus of MSCs took bluish-purple colour.

Fluorescent staining of rBMSC-scaffold construct revealed that the live cells emitted green fluorescence (Figure 2). On day 3 post seeding, no dead cells were seen.

SEM observation of the seeded scaffold on day 3 post-seeding showed stem cell attachment on the surface of HASi (Figure 3A). They had a wavy surface and the attachment was close but not tight. On day 17 post-seeding, the attached stem cells showed expansion and proliferation to form cell sheet-like structure with strong anchoring 
to the scaffold surface by footpads (Figure 3B). There was matrix deposition in the form of crystals over and around the attached stem cells (Figure 3C). The cells were seen closely attached to the side wall of the pores. The panoramic view of calcium matrix deposition by the attached cells was similar to snow covered caves (Figure 3D). The adjacent crystals physically entangled to form a strong interconnected network that showed similarity with calcium orthophosphate (Figure $3 \mathrm{E})$. The control HASi maintained in same culture conditions for the same time period as 17 days post-seeding samples, did not show any crystal deposits (Figure 3F).

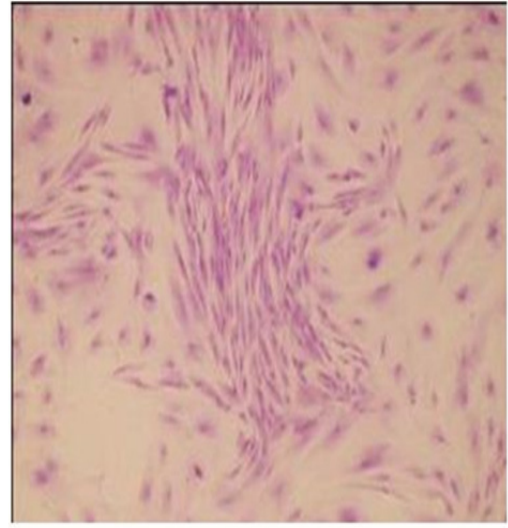

Figure IA Crystal violet staining of P3 rBMSC.

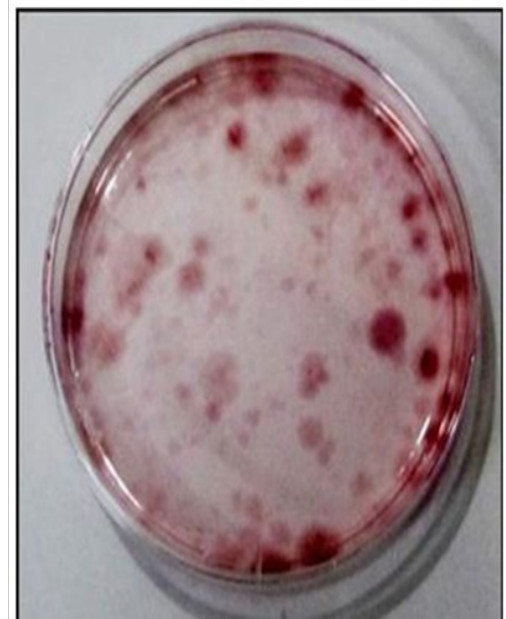

Figure IB Colony forming units of osteoblasts.

\section{CFU-ASSAY}

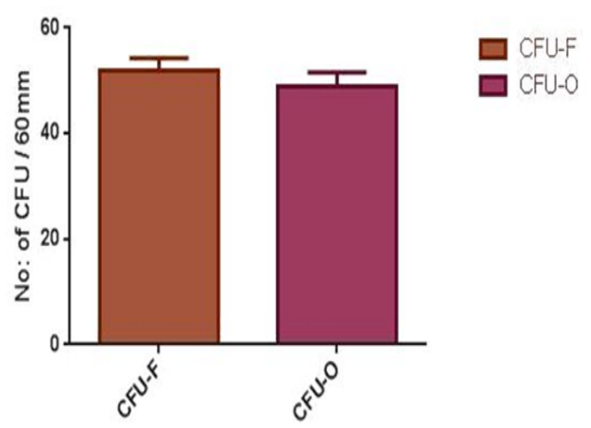

Figure IC Histogram showing number of CFU of fibroblasts and osteoblasts (mean $\pm \pm S D$ ) from an initial concentration of $1 \times 106$ cells.

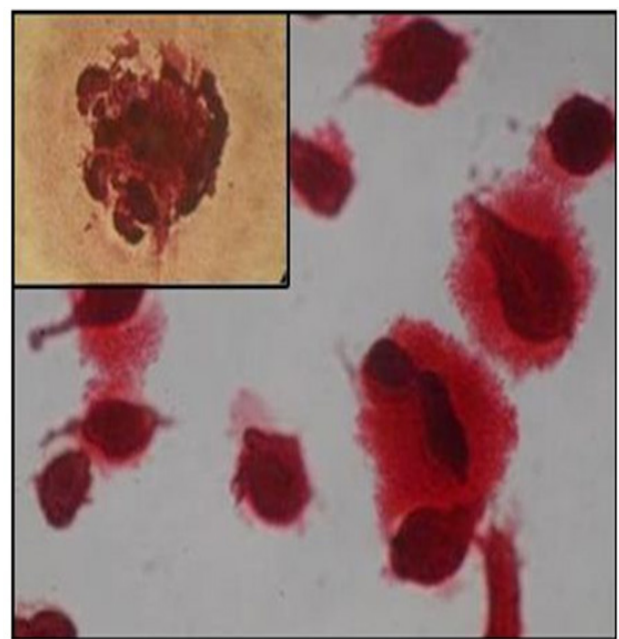

Figure ID Orange red staining calcium nodules representing osteoblastic differentiation of rBMSC (200X, 400X).

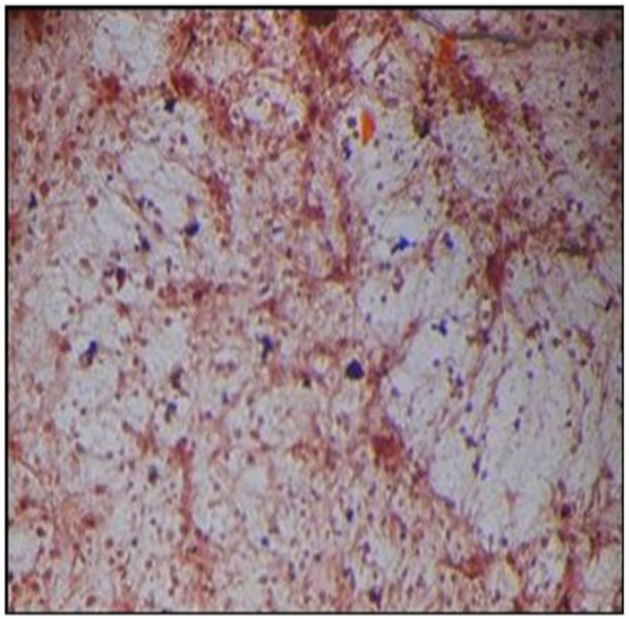

Figure IE Osteoblast with bluish purple nucleus after ALP staining (200X).

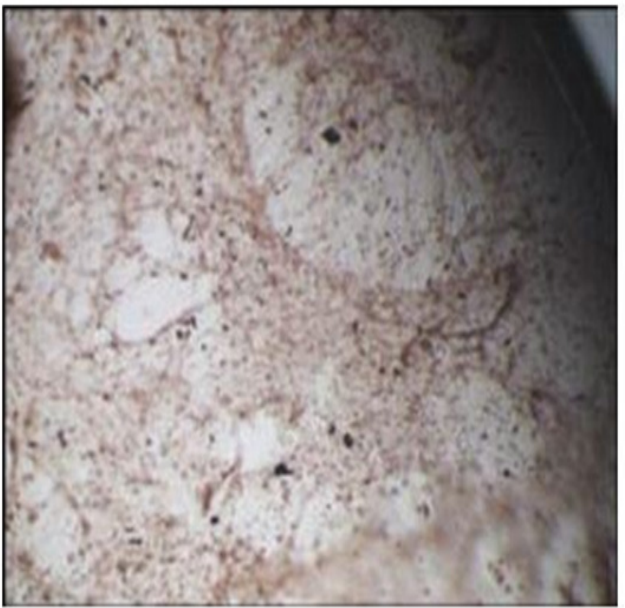

Figure IF Control well after ALP staining.

Trypsinization and subsequent alizarin red staining of the crystals deposits from day 17 post-seeding sample of HASi showed intense red staining indicative of presence of calcium (Figure 4). The 
histological sections stained with H\& E staining showed pink stained areas indicative of beginning of early new bone formation (Figure 5).

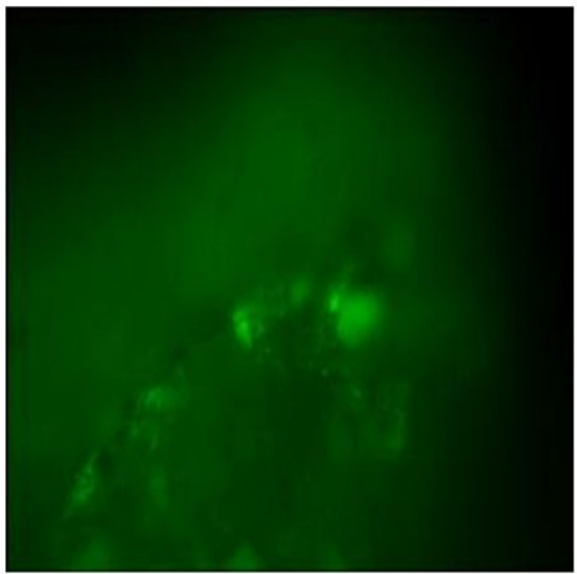

Figure 2 Live cells emitting green fluorescence on day 3 post-seeding on the HASi scaffold.

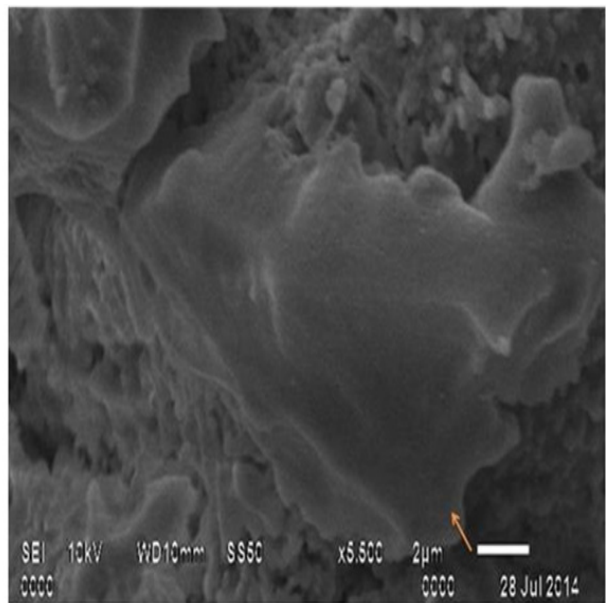

Figure 3A SEM analysis of rBMSC seeded HASi on day 3 showing attachment of stem cell (arrow).

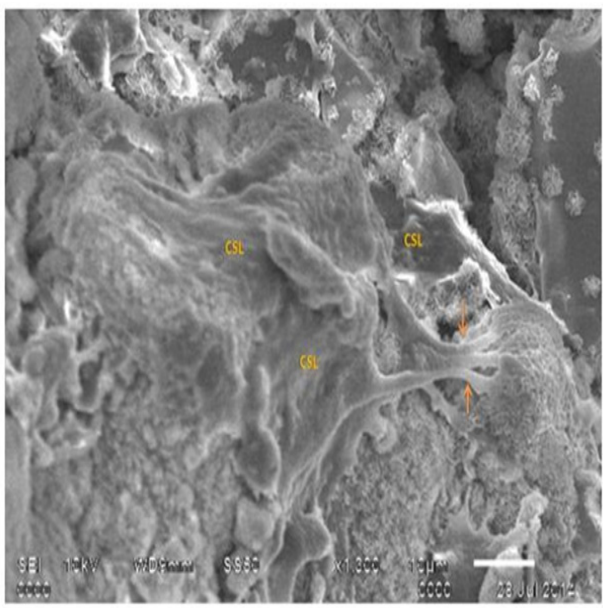

Figure 3B SEM analysis of rBMSC seeded HASi on day 17 post-seeding showing cell sheet like structure (CSL) strongly anchored to scaffold surface by footpads (arrows).

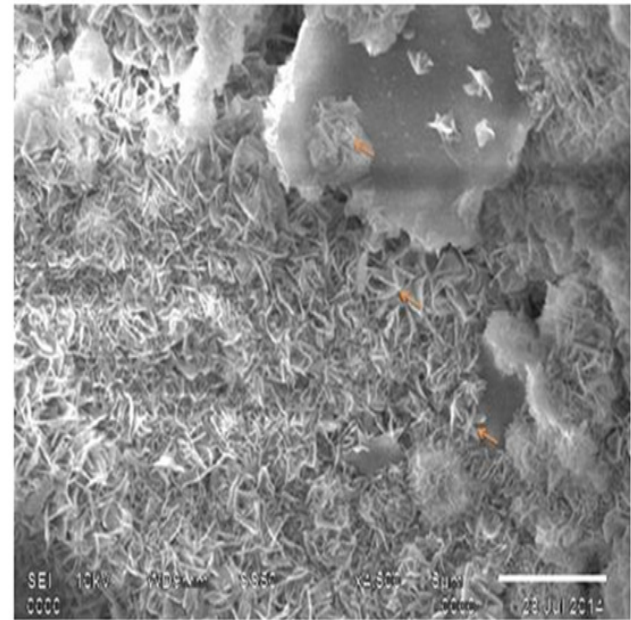

Figure 3C SEM analysis of rBMSC seeded HASi on day 17 post-seeding showing matrix deposition (arrows) over and around the attached cells.

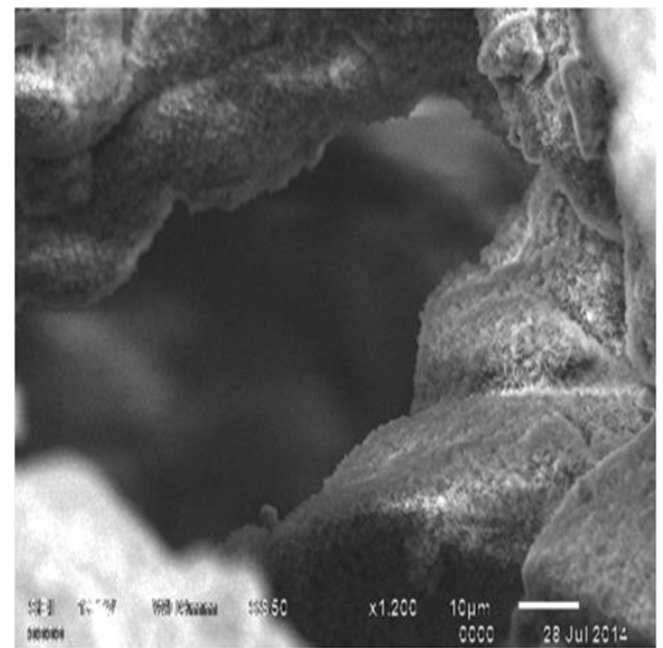

Figure 3D SEM analysis of rBMSC seeded HASi on day 17 post-seeding showing a panoramic view of matrix deposition over attached cells.

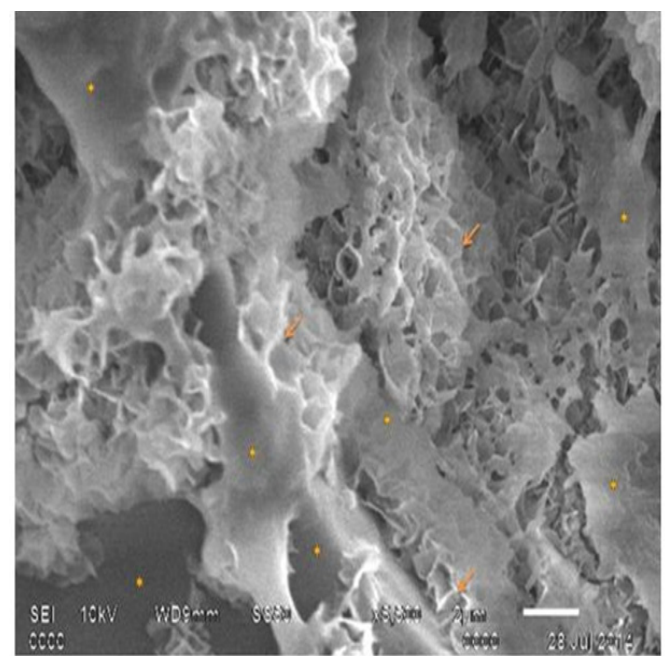

Figure 3E SEM analysis of a closer view of rBMSC seeded on HASi on day 17 post-seeding showing physical entanglement of calcium orthophosphate like crystals (arrows) over and around attached cells (dots). 


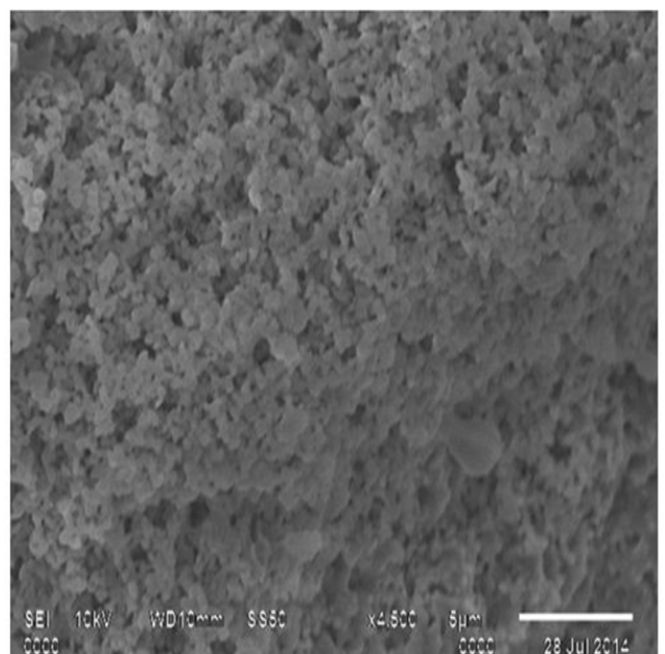

Figure 3F SEM analysis of the unseeded HASi after 17 days.

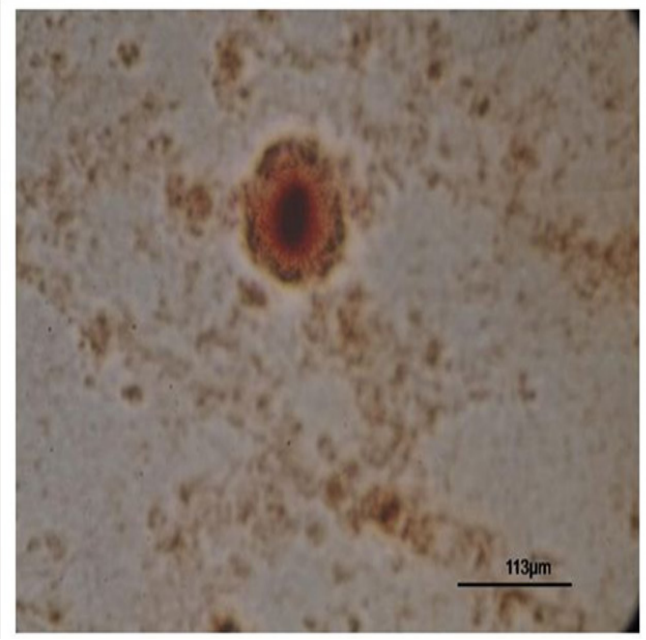

Figure 4 Light micrograph of alizarin red stained calcium crystal trypsinized from day 17 post-seeding HASi showing positive intense red colour indicating presence of calcium.

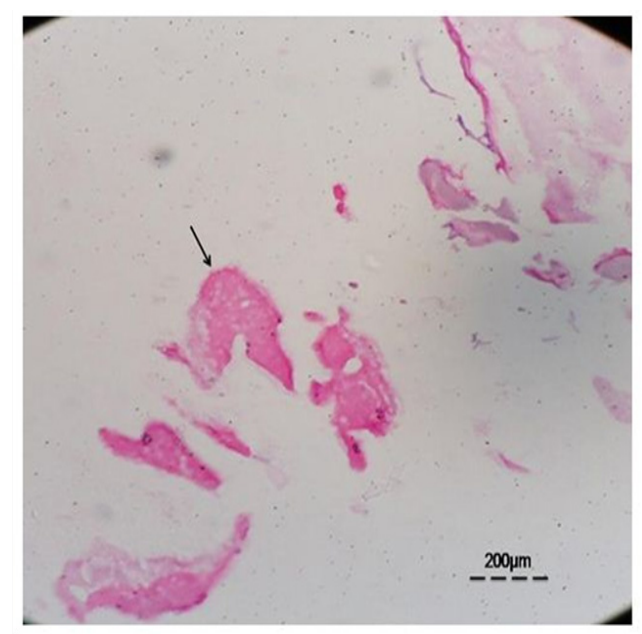

Figure 5 H \& E stained sections of decalcified day 17 post-seeded HASi scaffold showing dark pink stained areas (arrows) indicating early new bone formation.

\section{Discussion}

Scaffolds can potentially replace damaged or diseased tissues. They may be used for enhancing the in vivo viability and differentiating potential of stem cells. Hydroxyapatite is the most commonly used synthetic biomaterial for bone defects because of its osteoconductive properties, ability to integrate with bony tissue and minimal immune response but with the drawback of slow resorption rate. ${ }^{4,5}$ The faster resorption rate of silica coated hydroxyapatite is due to the greater dissolution of $\mathrm{Si}$ ions. ${ }^{7}$

Mesenchymal stem cells are known to have the ability to undergo osteogenic differentiation under suitable conditions. ${ }^{10}$ In this study the in vitro conditions for proliferation and differentiation of mesenchymal stem cells to osteoblastic lineage was established. Rabbit mesenchymal stem cells isolated from different compartments present different biological characteristics. The colony-forming efficiency of bone marrow derived rMSCs is higher than that of peripheral bloodderived rMSCs. ${ }^{11}$ Bone marrow-derived cells and periosteum-derived cells proliferate faster than do adipose - derived rMSCs. ${ }^{12}$ Fetal liverderived rMSCs present a higher adhesion capacity and proliferate faster than do adult bone marrow-derived $\mathrm{rMSCs},{ }^{13}$ but there are ethical regulations for its use.

Several procedures were proposed for simply harvesting autologous or homologous mesenchymal stem cells, expanding them in culture, inducing differentiation and seeding them on suitable scaffolds in accordance with the targeted tissue type and implanting the construct into the patient's body. ${ }^{14}$ Density gradient media is used most commonly for isolation of MSCs and their plastic adherent property was exploited for culture expansion. ${ }^{9}$ In this study, the cells reached $80-90 \%$ confluence by $10-12$ days following seeding in T25 flasks. The time period for reaching complete confluence by rBMSCs may be as long as $12-15$ days. ${ }^{9}$ In the present study, P1 to P3 passages showed uniform spindle shaped morphology which is in accordance with earlier reports. ${ }^{15}$

CFU-F and CFU-O assays revealed the proliferation capacity and colony forming characteristics of fibroblasts derived from rabbit bone marrow and osteoblasts from rBMSCs. CFU-F is recognized as the early osteoblastic cell precursors and the CFU-F assay is a useful method to enumerate the number of MSCs in bone marrow. ${ }^{16}$

Increase in the levels of the alkaline phosphatase activity, an intracellular enzyme necessary for mineralization is considered to be an early marker of cells oriented towards osteogenic production. It degrades inorganic pyrophosphate to release phosphate that is needed for mineralization. Adult MSC exposed to OS medium differentiate towards osteoblastic lineage..$^{17}$ Calcium deposits in the matrix were demonstrated by alizarin red S staining. This histological staining is based on the capacity of alizarin red to specifically stain matrix containing calcium and its positive appearance is considered an expression of bone matrix deposition. ${ }^{18}$ The co-localization of alizarin red stain with the deposit of amorphous material close to the nodular cell aggregates observed in OS cultures demonstrated that these amorphous deposits contain calcium and suggests that nodular cell aggregates are made up of cells committed to the osteoblastic lineage. In the present study, this target was reached in a simple and safe way using a cocktail of three different drugs, of which the key member was dexamethasone. Dexamethasone is necessary for in vitro bone nodule formation and mineralization in marrow derived cell cultures. The increase in ALP activity in OS culture is a marker of the commitment towards osteoblastic lineage. 
In vitro fluorescent staining of the seeded scaffold proved that there was attachment of mesenchymal stem cells on the HASi scaffold on day 3 post-seeding and these cells were alive. Fluorescent labeling of cells has been regarded as a powerful tool to determine the cell distribution in tissue constructs. ${ }^{19}$

SEM examination showed that on day 3 post-seeding, there was attachment of mesenchymal stem cells. Fibronectin used in the present study is a glycoprotein that gets adsorbed on the scaffold surface and serves as initial cell anchoring points. In addition, serum in the media is known to contain vitronectin in addition to fibronectin. The cells interact with these adsorbed proteins through integrins, especially, integrin-alpha-5-beta-1 in case of fibronectin, resulting in the activation of intracellular signal transduction cascades leading to adhesion complex formation and migration of cells. ${ }^{20}$ Morphology of mesenchymal stem cells varied from one substrate to another. The substrate characteristics that influence cell morphology include porosity, surface chemistry, bulk chemistry and surface finishing. ${ }^{21}$ By day 17 post-seeding, the morphology of attached stem cells was similar to a cell sheet-like structure with strong anchoring to the scaffold surface by footpads. Filopodia are cytoplasmic extensions by which osteoblasts and mesenchymal cells attach to the scaffold. The cytoplasm of adjacent Filopodia later zip-up to form flattened regions called foot pads or lamellipodia. However, the cell sheet-like morphology was noticed when goat bone marrow derived cells were seeded on the same HASi scaffold. ${ }^{22}$ The firm adhesion property of the cells may be due to the ability of silica coating that absorbs serum proteins. The expansion of cells and strong anchoring by footpads indicated that HASi scaffold promoted the proliferation and viability of rBMSC. The enhanced viability and proliferation of cells on HASi scaffold can be attributed to the multiphase composition (HA, tricalcium phosphate and calcium silicate) as well as silica content of HASi ${ }^{23}$ Similarly report suggested that silica-containing bioactive glass has significantly increased cell viability, proliferations, and differentiation of osteoblast derived from rat calvaria in an in vitro culture. $^{24}$

The clinical outcome of a seeded scaffold is inevitably decided by the number of live cells initially present which is in turn decided by the seeding technique and cell density used at the time of seeding. An ideal seeding method should ensure uniform distribution and infiltration of cells with minimum cell damage enabling cell viability, proliferation and distribution. Otherwise, localized cell attachment and growth in seeded scaffold would hamper the clinical application of large tissue engineered constructs. ${ }^{19}$ Surface seeding technique was used in the present study. Though it is ideal for surface distribution of cells, the cell attachment and crystal deposition inside the pores indicated the migration of mesenchymal cells into the pores, their proliferation, expansion and possible differentiation to osteogenic lineage. The sufficient seeding density and interconnected porous nature of the scaffold might have yielded these favorable results.

The microstructure of crystals as observed by SEM was similar to calcium orthophosphate. ${ }^{25}$ All mammalian calcified tissues are mainly formed of calcium orthophosphates. ${ }^{26}$ The presence of calcified matrix by day 17 post-seeding confirmed by positive alizarin staining of trypsinized crystals demonstrated either the osteogenic differentiation of attached stem cells owing to the osteoinductive property of the scaffold or surface dissolution resulting in super saturation of calcium and phosphate ions and their reprecipitation leading to the formation of a biological apatite layer, a property that allows the bioceramic to bond with the bone. However, the latter possibility is nullified by the absence of any such crystals in the unseeded scaffolds maintained in same culture conditions and time period as day 17 post-seeded test samples on SEM analysis and by evidence of early new bone formation in the histological sections. There are citations of bioceramic with osteoinductive properties. ${ }^{27}$ The ability of silica coated hydroxyapatite to promote osteoinduction in human bone marrow derived stromal cells is already recorded in literature. ${ }^{8}$ But, the cellular and biological mechanisms behind this remain unknown though the surface characteristics of the scaffold like high specific surface area resulting from concavities on the wall of macropores could be a possible reason. This property may also be in part due to the presence of silicon which is known to stimulate osteoblast proliferation and hence promote osteogenesis by direct control over the genes involved in cell cycle induction and progression ${ }^{28,29}$ and in part due to the peculiarities in the rBMSC-HASi interaction which requires further studies. This finding confirms the in vitro osteogenic induction potential of rBMSC-HASi construct when cultured in DMEM alone without any osteogenic induction media by 17 days post-seeding. Adhesion, proliferation and viability of hBMSC were found more in HASi compared to hydroxyapatite (HA). ${ }^{8}$ Similarly, proliferation, viability and osteogenic differentiation of goat BMSC was found more in HASi than HA scaffold in an in vitro culture system. ${ }^{23}$ Nevertheless, the ability of HASi in maintaining cell expansion and differentiation is a promising approach to address the problem of bone regeneration in older patients where more extensive in vitro expansion of smaller number of stem/progenitor cells is needed. ${ }^{8}$

\section{Conclusion and clinical relevance}

Bioscaffold HASi had the potential to induce osteogenic differentiation of rBMSCs resulting in calcium matrix deposition in the absence of osteogenic induction medium. The rBMSC-HASi construct may be a promising bone substitute for autografts in nonhealing and delayed fracture healing with potential bone loss. Further research is needed for understanding the cellular and molecular mechanisms of osteoinduction in this stem cell construct noticed in our study. Further research is also emphasized to investigate individual cell-scaffold combinations and investigate proliferation, viability and osteogenic differentiation of BMSC of different animal species on HASi scaffold for one ideal stem cell-based tissue engineering.

\section{Acknowledgements}

Authors are highly thankful to Prof. H. Varma, Sree Chitra Tirunal Institute for Medical Sciences and Technology, Trivandrum, Kerala (India) for providing the HASi scaffold.

\section{Conflict of interest}

The author declares no conflict of interest.

\section{References}

1. Lutolf MP, Hubbell JA. Synthetic biomaterials as instructive extracellular microenvironments for morphogenesis in tissue engineering. Nat Biotechnol. 2005;23(1):47-55.

2. Takezawa TA. A strategy for the development of tissue engineering scaffolds that regulate cell behavior. Biomaterials. 2003;24(13):22672275 .

3. Neuss S, Apel C, Buttler P, et al. Assessment of stem cell/biomaterial combinations for stem cell-based tissue engineering. Biomaterials. 2008;29(3):302-313. 
4. Neo M, Kotani S, Nakamura T, et al. A comparative study of ultra structure of the interfaces between four kinds of surface-active ceramic and bone. J Biomed Mater Res. 1992;26(11):1419-1432.

5. Duchyene P, Qiu Q. Bioactive ceramics: the effect of surface reactivity on bone formation and bone cell function. Biomaterials. 1999;29(2324):2287-2303

6. Vuola J, Taurio R, Goransson H, et al. Compressive strength of calcium carbonate and hydroxyapatite implants after bone-marrow-induced osteogenesis. Biomaterials. 1998;19(1-3):223-227.

7. EI Ghannam AR. Advanced bioceramic composite for bone tissue engineering: design principles and structure-bioactivity relationship. $J$ Biomed Mater Res A. 2004;69(3):490-501.

8. Nair MB, Bernhardt A, Lode A, et al. A bioactive triphasic ceramiccoated hydroxyapatite promotes proliferation and osteogenic differentiation of human bone marrow stromal cells. J Biomed Mater Res A. 2009;90(2):533-542.

9. Maiti SK, Shivkumar MU, Srivastava L, et al. Isolation, proliferation and morphological characteristics of bone marrow derived mesenchymal stem cells (BM-MSC) of different species of animals. Trends Biomater and Artif Organs. 2013;27(1):29-35.

10. Birmingham E, Niebur GL, McHugh PE, et al. Osteogenic differentiation of mesenchymal stem cells is regulated by osteocyte and osteoblast cells in a simplified bone niche. Eur Cell Mater. 2012;23:13-27.

11. Wan C, He Q, Li G. Allogenic peripheral blood derived mesenchyma stem cells (MSCs) enhance bone regeneration in rabbit ulna criticalsized bone defect model. J Orthop Res. 2006;24(4):610-618.

12. Hui JHP, Li L, Teo YH, et al. Comparative study of the ability of mesenchymal stem cells derived from bone marrow, periosteum and adipose tissue in treatment of partial growth arrest in rabbit. Tissue Eng. 2005;11(5-6):904-912.

13. Moreno R, Martinez GI, Rosal M, et al. Characterization of mesenchymal stem cells isolated from the rabbit fetal liver. Stem Cell Dev. 2010;19(10):1579-1588.

14. Dominici M, Le Blanc K, Mueller I, et al. Minimal criteria for defining multipotent mesenchymal stromal cells. The International Society for Cellular Therapy position statement. Cytotherapy. 2006;8(4):315-317.

15. Fan Z, Lu Y, Deng L, et al. Placenta-versus bone-marrow-derived mesenchymal cells for the repair of segmental bone defects in a rabbit model. FEBS J. 2012;279(13):2455-2465.
16. Simmons PJ, Torok Storb B. Identification of stromal cell precursors in human bone marrow by a novel monoclonal antibody, STRO-1. Blood. 1991;78(1):55-62.

17. Aubin JE, Liu F, Malaval L, et al. Osteoblast and chondroblast differentiation. Bone. 1995;17(2 Suppl):S77-S83.

18. Gregory CA, Gunn WJ, Peister A, et al. An alizarin red-based assay of mineralization by adherent in culture: comparison with cetylpyridinum chloride extraction. Anal Biochem. 2004;329(1):77-84.

19. Thevenot P, Nair A, Dey J, et al. Method to analyze three-dimensional cell distribution and infiltration in degradable scaffolds. Tissue Eng Part C Methods. 2008;14(4):319-331.

20. Assmann A, Delfs C, Munakata H, et al. Acceleration of autologous in vivo recellularization of decellularized aortic conduits by fibronectin surface coating. Biomaterials. 2013;34(25):6015-6026.

21. Annaz B, Hing KA, Kayser M, et al. Porosity variation in hydroxyapatite and osteoblast morphology:a scanning electron microscopy study. $J$ Microsc. 2004;215(Pt 1):100-110.

22. Nair MB, Babu SS, Varma HK, et al. A triphasic ceramic-coated porous hydroxyapatite for tissue engineering application. Acta Biomater. 2008;4(1):173-181.

23. Nair MB, Varma HK, John A. Triphasic ceramic coated hydroxyapatite as a niche for goat stem cell derived osteoblasts for bone regeneration and repair. J Mater Sci Mater Med. 2009;20(Suppl 1):S251-S258.

24. Valerio P, Guimaraes MH, Pereira MM, et al. Primary osteoblast cell response to sol-gel derived bioactive glass foams. J Mater Sci Mater Med. 2005;16(9):851-856.

25. Dorozhkin SV. Self-setting calcium orthophosphate formulations: cements, concretes, pastes and putties. J Mater Chem. 2011;1(1):1-48.

26. Bassett Jr H. Beiträge zum Studium der Calciumphosphate. III. Das System $\mathrm{CaO}-\mathrm{P}_{2} \mathrm{O}_{5}-\mathrm{H}_{2}$ O. Z. Anorg Chem. 1908;59:1-55.

27. Lobo SE, Arinzeh TL. Biphasic calcium phosphate ceramics for bone regeneration and tissue engineering applications. Materials. 2010;3(2):815-826

28. Zhang J, Zhou H, Yang K, et al. RhBMP-2-loaded calcium silicate/ calcium phosphate cement scaffold with hierarchically porous structure for enhanced bone tissue regeneration. Biomaterials. 2013;34(37):93819392. 